\title{
Accurate Analytic Approximations For Real-Time Specular Area Lighting
}

\author{
Pascal Lecocq
}

Gaël Sourimant

Jean-Eudes Marvie

Technicolor *
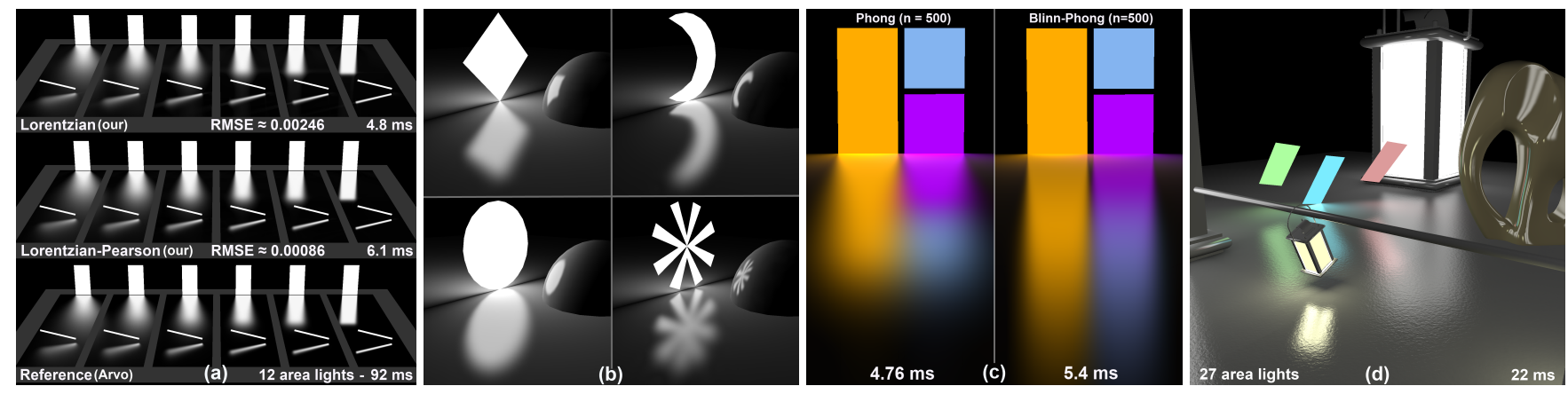

Figure 1: Our analytic approximations provide real-time performances to specular area lighting at a quality close to the ground truth (a,d). We support polygonal light source shapes of any kind (b), animated or not, and provide extension for half-vector based BRDFs (c).

\begin{abstract}
Accurate real-time rendering of specular surfaces is a challenging task when considering area light source illumination. The difficulty resides in the evaluation of a surface integral for which no practical solution exists except using expensive Monte Carlo methods. Recent techniques like the most representative point approach [Drobot 2014] alleviate this problem but make some accuracy trade-off to achieve real-time performance. We introduce analytic approximations for accurate real-time rendering of specular surfaces illuminated by polygonal light sources. Our solution is based on a reformulation of the contour integral [Arvo 1995] we approximate analytically with simple peak functions. In addition, using simple geometric operations, we extend the solution to handle more physically plausible BRDFs. Our solution works without any assumption on light source shape nor surface roughness, bringing real-time performances with a quality close to the ground truth.
\end{abstract}

\section{Closed form analytic approximations}

Prior work. Our analytic approximations build upon the contour integration method developed by [Arvo 1995] for Phong surfaces. The surface integral is evaluated by 1D angular integration over boundary edges of a polygonal light source. Each edge integral corresponds to a sum of integrals of simpler form with a closed solution. The length of the sum is parameterized by the shininess $n$, leading to $O(n)$ time computation per edge. Implemented on a GPU, such evaluation has severe impact on performances when $n>20$, limiting its usage to weakly glossy surfaces.

Our approach. We propose accurate analytic approximations that reduce the costly edge integration to $O(1)$ time whatever the surface roughness. We first reformulate the edge integral and identify a geometric series allowing us to cancel out the sum and reduce it to a single function $I$ to integrate. Even though no anti-derivative exist for $I$, an accurate analytic approximation can be obtained. We observe that the shape of $I$ roughly corresponds to a peak function with varying scale and width depending on $n$ and a geometric term $c$ (figure 2 left). Following this statement, we approximate $I$ using a single or combined peak shape functions $P$ having simple anti-derivative for constant time evaluation. The peak function $P$ is parameterized using an empirical prediction of the half width of $I$ and one point evaluation on $I$ using our reformulation. The simplest approximation is obtained using a Lorentzian peak function,

\footnotetext{
*\{pascal.lecocq, gael.sourimant, jean-eudes.marvie\} @ technicolor.com
}
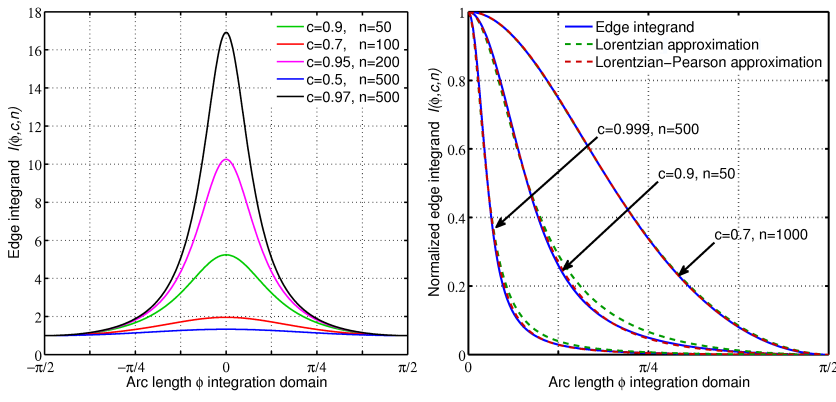

Figure 2: The edge integrand I reveals peak functions with various shapes (left) that can be fairly well approximated using simple and integrable peak shape functions (right).

reducing the integration to a single arctangent evaluation. A linear combination of a Lorentzian and a Pearson VII function provides the best match for integrand $I$ using a second fitting point in the tail of $I$ (figure 2 right). Unlike Chebychev or Fourier approximation, our solution is bound to one or a maximum of 2 analytic functions and does not suffer from any ringing artifacts.

Results. We tested our solution on an NVIDIA GPU GeForce GTX580 at a resolution of $1280 \times 720$ pixels, with full screen coverage and edge clipping for correct horizon handling. The Lorentzian approximation achieves the best performance $(\approx 0.23 \mathrm{~ms} /$ edge and $\mathrm{RMSE} \approx 0.00246$ ), while the Lorentzian-Pearson is the most accurate with unnoticeable difference with the ground truth (RMSE $\approx$ 0.00086 and $\approx 0.42 \mathrm{~ms} /$ edge).

Extension to other BRDFs. We extend the solution to handle half vector based BRDFs and remove the Phong restriction. We consider simple geometric operations on the hemisphere combined with an edge splitting strategy to compensate for the edge distortion introduced by the half vector transformation. As a result, we render plausible BRDFs in real-time with only a small computational overhead $(\approx 0.53 \mathrm{~ms} /$ edge with Lorentzian-Pearson $)$.

\section{References}

ARVO, J. 1995. Applications of irradiance tensors to the simulation of non-lambertian phenomena. In SIGGRAPH'95, 335-342.

Drobot, M. 2014. Physically based area lights. In GPU Pro 5, $67-100$. 